\title{
RESISTÊNCIA DE Bidens subalternans AOS HERBICIDAS INIBIDORES DA Enzima ACEtolactato Sintase Utilizados na Cultura da Soj $A^{1}$
}

\author{
Resistance of Bidens subalternans to the Acetolactate Synthase Inhibitor Herbicides Used in \\ Soybean Crop
}

\author{
GELMINI, G.A. ${ }^{2}$, VICTÓRIA FILHO, R. ${ }^{3}$, NOVO, M.C.S.S. ${ }^{4}$ e ADORYAN, M.L. ${ }^{5}$
}

\begin{abstract}
RESUMO - O uso contínuo e prolongado de produtos com o mesmo mecanismo de ação pode provocar a manifestação de biótipos resistentes. Para verificar possíveis novos casos de resistência, bem como alternativas para prevenção e manejo, foram coletadas sementes de Bidens subalternans na região de São Gabriel D' Oeste-MS, em plantas que sobreviveram a tratamentos em que inibidores da ALS foram sistematicamente utilizados. Em experimento conduzido em vasos em casa de vegetação, o biótipo com histórico de resistente foi comparado ao suscetivel quando submetido aos diversos herbicidas com diferentes mecanismos de ação usados em pós-emergência, os quais foram aplicados nas doses de zero, uma, duas, quatro e oito vezes a recomendada. Decorridos 20 dias, foram avaliadas a porcentagem de controle e a produção da fitomassa verde, visando estabelecimento de curvas de dose-resposta e obtenção dos fatores de resistência. O biótipo oriundo de área com histórico de aplicações repetidas de inibidores da ALS apresentou elevado nível de resistência aos herbicidas chlorimuron-ethyl e imazethapyr, demonstrando ser portador de resistência cruzada aos inibidores da ALS dos grupos das sulfoniluréias e imidazolinonas. Entretanto, esse biótipo foi eficientemente controlado pelos herbicidas fomesafen, lactofen, bentazon, glufosinato de amônio e glyphosate.
\end{abstract}

Palavras-chave: resistência cruzada, sulfoniluréia, imidazolinona.

\begin{abstract}
The continuous and prolonged use of products with the same mechanism of action can provoke the manifestation of resistant biotypes. In horder to verify possible new cases, as well as alternatives for prevention and control, seeds of Bidens subalternans were collected at São Gabriel D' Oeste (MS) region at plants that survived continuous treatments which sistematically ALS inhibitors. Through an experiment performed in pots inside a greenhouse, a resistant biotype was compared to a susceptible one when submitted to herbicides with different mechanisms of action and applied at post emergence. These herbicides were applied at doses zero, one, two, four and eight times the recommended dosage. Twenty days after, the control and the green weight production were analysed aiming to get the dose-response curves as well as the resistance factor. The biotype from the area with repeated application of ALS inhibitors showed a high level of resistance to chlorimuron-ethyl and imazethapyr, demonstrating therefore to be a carrier of crossed resistance to the ALS inhibitors of the sulfonilurea and imidazolinona groups. However, this biotype was controlled by fomesafen, lactofen, bentazon, ammonium glufosinate and glyphosate.
\end{abstract}

Key words: crossed resistance, sulfunylurea, imidazolinone.

1 Recebido para publicação em 24/1/2001 e na forma revisada em 7/8/2002.

Parte da tese do primeiro autor apresentada à ESALQIUSP, para obtenção do título de Doutor.

2 Doutorando do Departamento de Produção Vegetal da Escola Superior de Agricultura "Luiz de Queiroz" da Universidade de São Paulo - ESALQ/USP, Caixa Postal 09, 13419-900 Piracicaba-SP; ${ }^{3}$ Departamento de Produção Vegetal da ESALQ/USP. ${ }^{4}$ Centro de Ecofisiologia e Biofísica do Instituto Agronômico, Caixa Postal 28, 13001-970 Campinas-SP. ${ }^{5}$ Aventis CropScience do Brasil Ltda. Estação Agrícola Experimental, Caixa Postal 7, 13140-000 Paulínia-SP. 


\section{INTRODUÇÃO}

A cultura da soja constitui-se no agronegócio com maior capacidade de geração de divisas internacionais para o Brasil - por meio da exportação do complexo grão-óleo-farelo - e impulsiona diversos complexos agroindustriais, com relevante papel socioeconômico (Câmara, 1998). A produção brasileira de soja representa aproximadamente $20 \%$ da mundial. Possiveis acréscimos na oferta de grãos desta cultura no Brasil poderão ser obtidos não só pelo aumento da área de cultivo, mas também através de incrementos na sua produtividade (Barbosa et al., 1999). Dentre os componentes do sistema de produção, o adequado manejo das plantas daninhas para minimizar os efeitos da interferência indesejável destas sobre a cultura constitui importante prática para obtenção de maiores rendimentos por unidade de área (Durigan et al., 1983; Pitelli, 1985; Melhorança et al., 1996).

Embora o controle das plantas daninhas possa ser feito pelos métodos cultural, biológico, físico, mecânico e químico, em se tratando de cultura típica de médias e grandes propriedades, o método químico vem sendo amplamente adotado, em razão da pronta disponibilidade de herbicidas e da economia de mão-de-obra, além da rapidez da aplicação (Burnside, 1992; Melo Filho et al., 1996; EMBRAPA, 1998). Dentre os herbicidas atualmente disponiveis para a cultura da soja, os inibidores da acetolactato sintase (ALS) aparecem com grande destaque, não só em virtude do número de opções voltadas para essa cultura, como também pela eficiência apresentada no controle das principais espécies infestantes (EMBRAPA, 1998; Rodrigues \& Almeida, 1998).

No entanto, as constantes e repetidas aplicações desses herbicidas têm exercido grande pressão de seleção, reduzindo ou eliminando a população suscetível, possibilitando assim o aumento de espécies tolerantes ou a manifestação de biótipos resistentes que provavelmente já existiam na comunidade, porém em freqüência bastante baixa (Holt \& Le Baron, 1990; Vidal \& Fleck, 1997).

Até recentemente, a ocorrência de Bidens subalternans era pouco mencionada, provavelmente devido ao fato de a espécie ser bastante semelhante a Bidens pilosa, da qual se diferencia, entre outros, pelo número de aristas dos aquênios (Kissmann \& Groth, 1999). Entretanto, sua presença tem aumentado significativamente em diversas áreas, e no Mato Grosso e norte do Mato Grosso do Sul, em muitas propriedades, sua infestação apresenta-se maior que a de B. pilosa (Sanches \& Zandonade, 1997); já no Paraná sua ocorrência foi verificada em todas as regiões produtoras de soja do estado (Gazziero et al., 1998). Com base na legislação que disciplina produtos agrotóxicos e afins, não existem atualmente indicações de herbicidas para o seu controle (Rodrigues \& Almeida, 1998). Também, conforme pode ser observado nas diversas recomendações técnicas existentes para a cultura da soja, a espécie ainda não se encontra relacionada entre as plantas daninhas para as quais estão disponíveis indicações de controle com herbicidas (EMBRAPA, 1998; Pereira et al., 1999). Kissmann \& Groth (1999) a consideram um pouco menos sensível à ação de alguns produtos, requerendo dose maior que a necessária para controlar B. pilosa. Este trabalho teve como objetivos determinar a manifestação de resistência em biótipos provenientes do Mato Grosso do Sul, estabelecer as doses necessárias para provocar o mesmo efeito em plantas suscetiveis e resistentes e estabelecer alternativas para o controle químico.

\section{MATERIAL E MÉTODOS}

O experimento foi conduzido em casa de vegetação na Estação Experimental da AgrEvo, localizada no município de Cosmópolis-SP, utilizando-se sementes de $B$. subalternans coletadas no final da safra 1996/97, colhidas de plantas que sobreviveram a tratamentos sistemáticos com diversos inibidores da ALS em área produtora de soja da região de São Gabriel D’Oeste-MS. Para efeito de comparação, foram também coletadas sementes de biótipo suscetível, de áreas nunca tratadas com inibidores da ALS, que foram secas ao ar, limpas para a retirada da terra e impurezas, devidamente acondicionadas em recipientes e armazenadas em câmara refrigerada $\left(9{ }^{\circ} \mathrm{C}\right)$ com controle de umidade (25\%), de modo a possibilitar sua preservação. Para o plantio, foram utilizados vasos plásticos perfurados, com capacidade para $1,3 \mathrm{~L}$, medindo $14,8 \mathrm{~cm}$ no diâmetro maior, $9,8 \mathrm{~cm}$ no menor e $12,2 \mathrm{~cm}$ de 
altura (vaso $\mathrm{n}^{\circ}$ 15), que receberam substrato composto por três partes de terra para uma de húmus, o qual apresentou $33,4 \%$ de areia grossa, $11,8 \%$ de areia fina, 10,2\% de argila e 18,9\% de matéria orgânica.

A semeadura foi efetuada em 17/11/98, colocando-se dez sementes em cada vaso. Após a germinação, procedeu-se ao desbaste, deixando quatro plantas por vaso, que receberam irrigação por aspersão através de equipamento automático, sempre que necessário, para manter o substrato com adequado nível de umidade.

Os tratamentos foram dispostos em blocos ao acaso com quatro repetições, envolvendo biótipos de $B$. subalternans, e doses de herbicidas de aplicação em pós-emergência que apresentam diferentes mecanismos de ação. Foram testados chlorimuron-ethyl a 0, 15, 30, 60 e $120 \mathrm{~g} \mathrm{ha}^{-1}$; imazethaphyr a $0,100,200,400 \mathrm{e}$ $800 \mathrm{~g} \mathrm{ha}^{-1}$; fomesafen a $0,250,500,1.000 \mathrm{e}$ $2.000 \mathrm{~g} \mathrm{ha}^{-1}$; lactofen a 0, 120, 240, $480 \mathrm{e}$ $960 \mathrm{~g} \mathrm{ha}^{-1}$; bentazon a $0,720,1.440,2.880 \mathrm{e}$ $5.760 \mathrm{~g} \mathrm{ha}^{-1}$; glufosinato de amônio a 0, 150, 300,600 e $1.200 \mathrm{~g} \mathrm{ha}^{-1}$; e glyphosate a 0,180 , 360, 720 e $1.440 \mathrm{~g} \mathrm{ha}^{-1}$. Nos tratamentos com chlorimuron-ethyl e bentazon foi adicionado óleo mineral emulsionável a $0,05 \%$ e $0,5 \%$ (v/v) às respectivas caldas de pulverização, enquanto o adjuvante nonil fenol oxietilado e álcool isopropílico a $0,2 \%(\mathrm{v} / \mathrm{v})$ foram adicionados às caldas de fomesafen e glufosinato de amônio. Os herbicidas foram aplicados em 12/12/98, quando as plantas apresentavam duas a quatro folhas, empregando-se pulverizador de precisão, pressurizado, tendo como gás propelente o ar comprimido e equipado com bicos de jato plano ("leque") XR110015. A pressão de trabalho de 2,5 bar possibilitou consumo de calda equivalente a $200 \mathrm{~L} \mathrm{ha}^{-1}$.

Vinte dias após as aplicações dos tratamentos, avaliou-se diretamente a porcentagem de controle, atribuindo-se para ausência de injúria o valor zero e para a morte da planta o valor 100 , de modo a obter posteriormente a dose que exerceu $50 \%$ de controle $\left(\mathrm{DC}_{50}\right)$. Nessa mesma época, as plantas foram cortadas rente ao solo e pesadas, a fim de que fosse obtida a fitomassa verde da parte aérea, cujos valores foram transformados em porcentagem de produção em relação à testemunha e utilizados para a determinação da dose que reduziu 50\% a mesma $\left(\mathrm{GR}_{50}\right)$. Os dados foram submetidos à análise de variância, utilizando-se o teste $\mathrm{F}$ para verificar a homogeneidade entre tratamentos com herbicida, dose, biótipo e suas interações, e, quando expressos em porcentagem, transformados em arc sen $(\mathrm{x} / 100)^{1 / 2}$. Quando significativos, compararam-se médias dos fatores qualitativos e suas interações por meio do teste de Tukey a 5\%. Para cada biótipo, a análise de doses de herbicida foi efetuada pelo programa de ajuste "Curve Expert 1.3". Cada conjunto foi caracterizado através da equação que melhor representou o comportamento da variável dentro de cada biótipo em relação ao efeito dos tratamentos com os herbicidas e suas doses, onde os coeficientes de determinação $\left(\mathrm{r}^{2}\right)$ foram superiores a 0,96 , sendo discutidos apenas os desdobramentos em que o teste $\mathrm{F}$ foi significativo. As equações de ajuste permitiram o cálculo da dose que provocou $50 \%$ de controle ou da produção de fitomassa verde em relação à testemunha. A partir desses valores foi determinado o fator de resistência, que representa o número de vezes que a dose necessária para proporcionar $50 \%$ de controle ou da produção de fitomassa verde do biótipo suscetível deve ser superior, a fim de que possa ocorrer o mesmo efeito sobre o biótipo resistente.

\section{RESULTADOS E DISCUSSÃO}

Os valores encontrados mostraram que apenas tratamentos com chlorimuron-ethyl e imazethapyr não controlaram o biótipo com histórico de resistente, mesmo quando foram aplicadas doses oito vezes acima da recomendada (Tabela 1). Os índices de controle do biótipo resistente situaram-se muito abaixo do mínimo aceitável $(2,5$ e $31,2 \%)$ e dos verificados quando a população suscetível foi submetida a idênticos tratamentos (92,5 e 100\%), para chlorimuron-ethyl. Em se tratando do imazethapyr, os resultados foram semelhantes, ou seja, variaram entre 5,0 e 47,5\% para o biótipo resistente e 92,5 e $100 \%$ para o suscetível. O mesmo foi observado para porcentagem de fitomassa verde em relação à testemunha, visto que os valores obtidos no biótipo em estudo variaram entre 92,4 e $98,2 \%$ e 77,3 e $96,6 \%$, respectivamente nos tratamentos com chlorimuronethyl e imazethapyr. Já no suscetivel as plantas foram praticamente eliminadas, demonstrando que foi apropriadamente controlado por esses dois herbicidas (Tabela 1). 
Tabela 1 - Porcentagem de controle e produção de fitomassa verde epígea dos biótipos resistente (BR) e suscetível (BS) aos 20 dias após a aplicação dos herbicidas. Cosmópolis-SP, 1998

\begin{tabular}{|c|c|c|c|c|c|}
\hline \multirow{2}{*}{ Tratamento } & \multirow{2}{*}{$\begin{array}{c}\text { Dose } \\
\left(\mathrm{g} \mathrm{ha}^{-1}\right)\end{array}$} & \multicolumn{2}{|c|}{ Porcentagem de controle $^{1^{\prime}}$} & \multicolumn{2}{|c|}{ Fitomassa verde ${ }^{2 /}$} \\
\hline & & $\mathrm{BR}$ & BS & $\mathrm{BR}$ & $\mathrm{BS}$ \\
\hline \multirow{5}{*}{ chlorimuron-ethyl ${ }^{4 /}$} & 0 & $0,0 \mathrm{a}^{3 /}$ & $0,0 \mathrm{a}^{3 /}$ & $100,0 \mathrm{a}^{3 /}$ & $100,0 \mathrm{a}^{3 /}$ \\
\hline & 15 & $2,5 \mathrm{~b}$ & $92,5 \mathrm{a}$ & $98,2 \mathrm{a}$ & $29,8 \mathrm{~b}$ \\
\hline & 30 & $2,5 \mathrm{~b}$ & $97,0 \mathrm{a}$ & $97,4 \mathrm{a}$ & $25,0 \mathrm{~b}$ \\
\hline & 60 & $18,7 \mathrm{~b}$ & $100,0 \mathrm{a}$ & 93,6 a & $21,7 \mathrm{~b}$ \\
\hline & 120 & $31,2 \mathrm{~b}$ & $100,0 \mathrm{a}$ & $92,4 \mathrm{a}$ & $20,6 \mathrm{~b}$ \\
\hline \multirow{5}{*}{ imazethapyr } & 0 & $0,0 \mathrm{a}$ & $0,0 \mathrm{a}$ & $100,0 \mathrm{a}$ & $100,0 \mathrm{a}$ \\
\hline & 100 & $5,0 \mathrm{~b}$ & $92,5 \mathrm{a}$ & 96,6 a & $29,7 \mathrm{~b}$ \\
\hline & 200 & $8,7 \mathrm{~b}$ & $97,7 \mathrm{a}$ & $93,0 \mathrm{a}$ & $23,9 \mathrm{~b}$ \\
\hline & 400 & $31,2 \mathrm{~b}$ & $99,5 \mathrm{a}$ & $78,2 \mathrm{a}$ & $22,4 \mathrm{~b}$ \\
\hline & 800 & $47,5 \mathrm{~b}$ & $100,0 \mathrm{a}$ & $77,3 \mathrm{a}$ & $20,7 \mathrm{~b}$ \\
\hline \multirow{5}{*}{ fomesafen ${ }^{5 /}$} & 0 & $0,0 \mathrm{a}$ & $0,0 \mathrm{a}$ & $100,0 \mathrm{a}$ & $100,0 \mathrm{a}$ \\
\hline & 250 & 90,7 a & $89,5 \mathrm{a}$ & $39,5 \mathrm{a}$ & $39,6 \mathrm{a}$ \\
\hline & 500 & $99,0 \mathrm{a}$ & $99,5 \mathrm{a}$ & $28,4 \mathrm{a}$ & $24,4 \mathrm{a}$ \\
\hline & 1.000 & $100,0 \mathrm{a}$ & $100,0 \mathrm{a}$ & $25,5 \mathrm{a}$ & $26,1 \mathrm{a}$ \\
\hline & 2.000 & $100,0 \mathrm{a}$ & $100,0 \mathrm{a}$ & $22,4 \mathrm{a}$ & $22,2 \mathrm{a}$ \\
\hline \multirow{5}{*}{ lactofen } & 0 & $0,0 \mathrm{a}$ & $0,0 \mathrm{a}$ & $100,0 \mathrm{a}$ & $100,0 \mathrm{a}$ \\
\hline & 120 & 96,5 a & $97,0 \mathrm{a}$ & $25,8 \mathrm{a}$ & $25,7 \mathrm{a}$ \\
\hline & 240 & $100,0 \mathrm{a}$ & $100,0 \mathrm{a}$ & $25,9 \mathrm{a}$ & $23,2 \mathrm{a}$ \\
\hline & 480 & $100,0 \mathrm{a}$ & $100,0 \mathrm{a}$ & $21,9 \mathrm{a}$ & $20,2 \mathrm{a}$ \\
\hline & 960 & $100,0 \mathrm{a}$ & $100,0 \mathrm{a}$ & $18,7 \mathrm{a}$ & $18,9 \mathrm{a}$ \\
\hline \multirow{5}{*}{ bentazon $^{6 /}$} & 0 & $0,0 \mathrm{a}$ & $0,0 \mathrm{a}$ & $100,0 \mathrm{a}$ & $100,0 \mathrm{a}$ \\
\hline & 720 & 83,7 a & 85,7 a & $35,9 \mathrm{a}$ & $36,3 \mathrm{a}$ \\
\hline & 1.440 & $96,5 \mathrm{a}$ & $97,2 \mathrm{a}$ & $26,1 \mathrm{a}$ & $26,9 \mathrm{a}$ \\
\hline & 2.880 & $100,0 \mathrm{a}$ & $100,0 \mathrm{a}$ & $21,4 \mathrm{a}$ & $21,8 \mathrm{a}$ \\
\hline & 5.760 & $100,0 \mathrm{a}$ & $100,0 \mathrm{a}$ & $19,0 \mathrm{a}$ & $17,9 \mathrm{a}$ \\
\hline \multirow{5}{*}{ glufosinato de amônio ${ }^{5 /}$} & 0 & $0,0 \mathrm{a}$ & $0,0 \mathrm{a}$ & $100,0 \mathrm{a}$ & $100,0 \mathrm{a}$ \\
\hline & 150 & 93,7 a & 93,7 a & $30,5 \mathrm{a}$ & $29,8 \mathrm{a}$ \\
\hline & 300 & $99,5 \mathrm{a}$ & $97,5 \mathrm{a}$ & $23,4 \mathrm{a}$ & $21,7 \mathrm{a}$ \\
\hline & 600 & $100,0 \mathrm{a}$ & $100,0 \mathrm{a}$ & $21,7 \mathrm{a}$ & $19,7 \mathrm{a}$ \\
\hline & 1.200 & $100,0 \mathrm{a}$ & $100,0 \mathrm{a}$ & $19,1 \mathrm{a}$ & $17,9 \mathrm{a}$ \\
\hline \multirow{5}{*}{ glyphosate } & 0 & $0,0 \mathrm{a}$ & $0,0 \mathrm{a}$ & $100,0 \mathrm{a}$ & $100,0 \mathrm{a}$ \\
\hline & 180 & 97,7 a & 97,7 a & $25,7 \mathrm{a}$ & $23,8 \mathrm{a}$ \\
\hline & 360 & $100,0 \mathrm{a}$ & $100,0 \mathrm{a}$ & $28,0 \mathrm{a}$ & $20,2 \mathrm{a}$ \\
\hline & 720 & $100,0 \mathrm{a}$ & $100,0 \mathrm{a}$ & $12,2 \mathrm{a}$ & $10,0 \mathrm{a}$ \\
\hline & 1.440 & $100,0 \mathrm{a}$ & $100,0 \mathrm{a}$ & $8,8 \mathrm{a}$ & $10,2 \mathrm{a}$ \\
\hline dms $5 \%$ & & \multirow{2}{*}{\multicolumn{2}{|c|}{$\begin{array}{l}2,20 \\
2,29\end{array}$}} & \multirow{2}{*}{\multicolumn{2}{|c|}{$\begin{array}{l}3,41 \\
5,29\end{array}$}} \\
\hline $\mathrm{CV}(\%)$ & & & & & \\
\hline
\end{tabular}

1/ Dados transformados em arco seno $(\mathrm{x} / 100)^{1 / 2}$ para análise; ${ }^{2 /}$ Produção de fitomassa verde em porcentagem, em relação à testemunha; ${ }^{3 /}$ Médias seguidas da mesma letra, dentro de cada dose para cada herbicida e que comparam os biótipos, não diferem entre si pelo teste de Tukey a $5 \% ;{ }^{4 /}$ Com a adição de óleo mineral emulsionável a $0,05 \%$ (v/v); ${ }^{5 /}$ Com a adição de nonil fenol oxietilado e álcool isopropílico a $0,2 \%(\mathrm{v} / \mathrm{v}) ;{ }^{6}$ Com a adição de óleo mineral emulsionável a $0,5 \%(\mathrm{v} / \mathrm{v})$. 
As curvas de dose-resposta para chlorimuron-ethyl e imazethapyr são apresentadas na Figura 1, e os valores da $\mathrm{DC}_{50}$ para chlorimuron-ethyl calculados a partir das equações de ajuste, indicam que estes se encontram acima da dose máxima aplicada (120 $\left.\mathrm{g} \mathrm{ha}^{-1}\right)$, enquanto para o suscetível foi de $8,7 \mathrm{~g} \mathrm{ha}^{-1}$. Em relação à fitomassa verde, observou-se, também para o biótipo em questão, a necessidade de doses superiores a $120 \mathrm{~g} \mathrm{ha}^{-1}$ para reduzir em $50 \%$ a produção, enquanto para o suscetivel foram necessários apenas $11,9 \mathrm{~g} \mathrm{ha}^{-1}$. Com base nesses resultados, constatou-se que, devido às doses elevadas, o fator de resistência foi superior a 22,0 e 86,4 , respectivamente, para porcentagem de controle e fitomassa verde epígea. Para imazethapyr, verificou-se que, para o biótipo resistente, a dose necessária para causar 50\% de controle e redução na fitomassa verde da planta deveria ser superior a $800 \mathrm{~g} \mathrm{ha}^{-1}$ enquanto no suscetivel seriam necessários 26,3 e $68,8 \mathrm{~g} \mathrm{ha}^{-1}$, respectivamente, para causar o mesmo efeito. Desse modo, os fatores de resistência para ambos os parâmetros analisados foram superiores a 26 (Tabela 2 ).

Essa situação evidencia a ocorrência de resistência cruzada aos inibidores da ALS representados para esses herbicidas pertencentes aos grupos das sulfoniluréias e imidazolinonas, conforme preconizado por Powles \& Howat (1990), Christoffoleti et al. (1994), Cortez \& Christoffoleti (1999) e Vargas et al. (1999). A ocorrência da resistência pode estar relacionada com a presença de genótipos que sobreviviam na comunidade original em níveis bastante baixos, como considerado por Guttieri et al. (1992), Vidal \& Fleck (1997) e Mattielo et al. (1999).
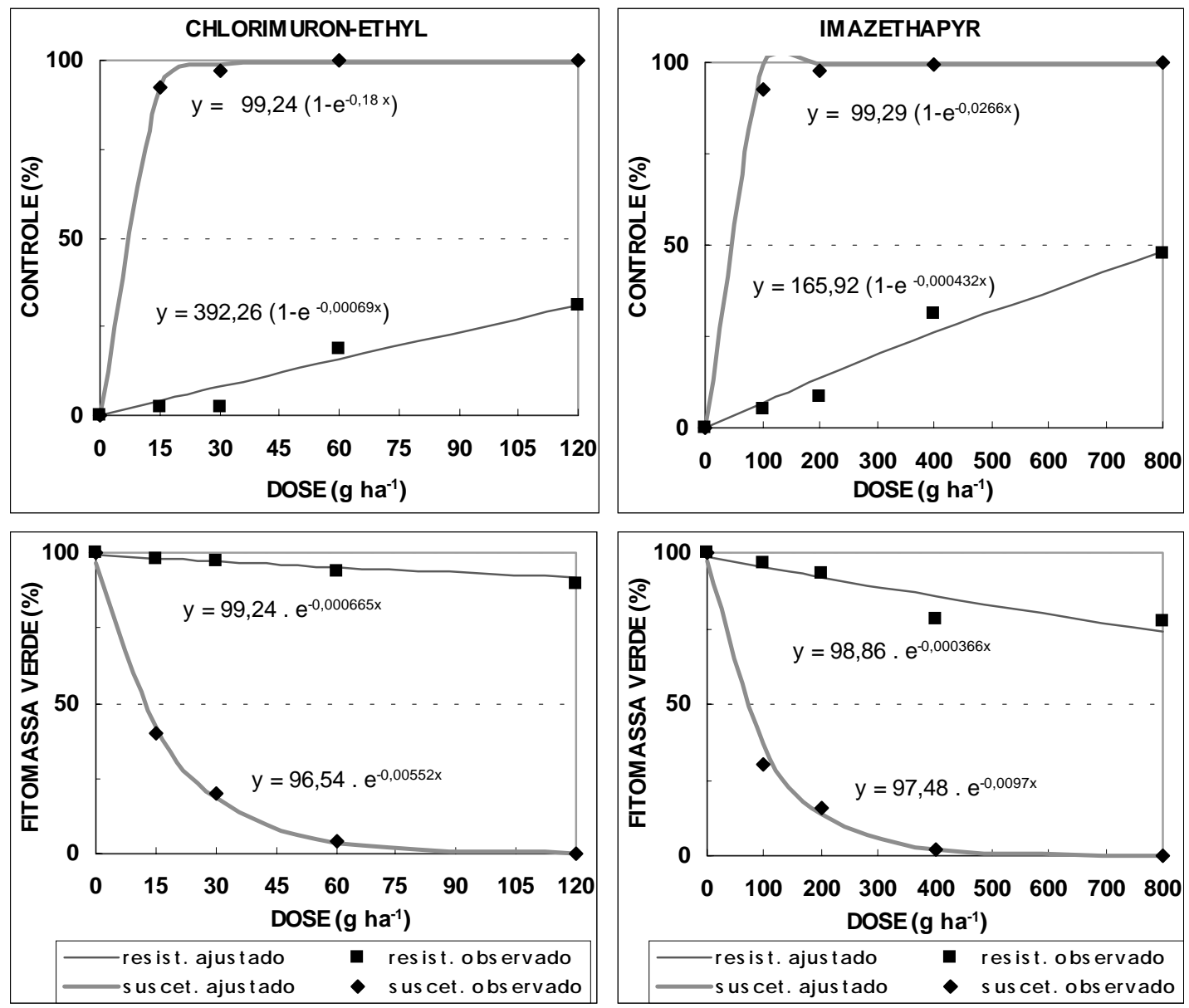

Figura 1 - Curvas de dose-resposta dos herbicidas chlorimuron-ethyl e imazethaphyr para Bidens subalternans (biótipos BR e BS). Cosmópolis-SP, 1998. 
Tabela 2 - Valores da $\mathrm{DC}_{50}, \mathrm{GR}_{50}$ e FR dos herbicidas chlorimuron-ethyl e imazethapyr referentes às porcentagens de controle e produção de fitomassa verde nos biótipos resistente (BR) e suscetível (BS). Cosmópolis-SP, 1998

\begin{tabular}{|c|c|c|c|c|c|}
\hline \multirow{2}{*}{ Biótipo } & \multicolumn{2}{|c|}{ Porcentagem de controle } & \multirow[t]{2}{*}{ Biótipo } & \multicolumn{2}{|c|}{ Fitomassa verde $(\%)$} \\
\hline & $\mathrm{DC}_{50}$ & FR & & $\mathrm{GR}_{50}$ & FR \\
\hline \multicolumn{6}{|c|}{ Chlorimuron-ethyl } \\
\hline $\mathrm{BR}$ & $>120$ & \multirow{2}{*}{$>22,00$} & $\mathrm{BR}$ & $>120$ & \multirow{2}{*}{$>86,48$} \\
\hline $\mathrm{BS}$ & 8,76 & & $\mathrm{BS}$ & 11,92 & \\
\hline \multicolumn{6}{|c|}{ Imazethapyr } \\
\hline $\mathrm{BR}$ & $>8,00$ & \multirow{2}{*}{$>26,00$} & $\mathrm{BR}$ & $>800$ & \multirow{2}{*}{$>27,05$} \\
\hline $\mathrm{BS}$ & 26,33 & & $\mathrm{BS}$ & 68,83 & \\
\hline
\end{tabular}

$\mathrm{DC}_{50}$ - dose que exerceu $50 \%$ de controle; $\mathrm{GR}_{50}$ - dose que reduziu em $50 \%$ a produção de fitomassa verde epígea; e FR - número de vezes em que a dose do herbicida necessária para proporcionar 50\% de controle ou produção de fitomassa verde epígea no biótipo suscetível deve ser superior, para que ocorra o mesmo efeito no biótipo resistente.

No entanto, quanto aos tratamentos com os demais herbicidas (Tabela 1), verificou-se que bentazon ( $\left.720 \mathrm{~g} \mathrm{ha}^{-1}\right)$, fomesafen $\left(250 \mathrm{~g} \mathrm{ha}^{-1}\right)$, lactofen (120 $\left.\mathrm{g} \mathrm{ha}^{-1}\right)$, glufosinato de amônio (150 $\mathrm{g} \mathrm{ha}^{-1}$ ) e glyphosate (180 $\mathrm{g} \mathrm{ha}^{-1}$ ) exerceram controle eficaz em ambos os biótipos. Desse modo, tornam-se importantes opções para controlar populações dessa espécie, mesmo quando formadas por biótipo resistente aos inibidores da ALS, como chlorimuron-ethyl e imazethapyr, evitando ainda os efeitos da seleção indesejável.

\section{LITERATURA CITADA}

BARBOSA, M. Z.; FERREIRA, C. R. R. P. T.; FREITAS, S. M. Sazonalidade de preços de exportação do complexo soja no Brasil. In: CONGRESSO BRASILEIRO DE SOJA, 1., Londrina, 1999. Anais... Londrina: EMBRAPA-Soja, 1999. p. 439.

BURNSIDE, O. C. Rationale for developing herbicideresistant crops. Weed Technol., v. 6, n. 3, p. 621-625, 1992.

CÂMARA, G. M. S. Desempenho produtivo dos cultivares de soja IAC-17, IAC-12 e IAC-19, semeados em três épocas de semeadura e em cinco densidades de plantas. Piracicaba: Escola Superior de Agricultura "Luiz de Queiroz", 1998. 165 p. Tese (Livre-Docência) - Escola Superior de Agricultura “Luiz de Queiroz”, 1998.

CHRISTOFFOLETI, P. J.; VICTORIA FILHO, R.; DA SILVA, C. B. Resistência de plantas daninhas aos herbicidas. Planta Daninha, v. 12, n. 1, p. 13-20, 1994.

CORTEZ, M. G.; CHRISTOFFOLETI, P. J. Mecanismos responsáveis pela resistência das plantas daninhas aos herbicidas. In: CURSO DE MANEJO DA RESISTÊNCIA DE PLANTAS DANINHAS AOS HERBICIDAS, 2., 1999, Ponta Grossa. Anais... Ponta Grossa, PR: AECG, 1999. p. $109-130$.
DURIGAN, J. C.; VICTORIA FILHO, R.; MATUO, T.; PITELLI, R.A. Período de matocompetição na cultura da soja (Glycine max (L.) Merril), cultivares Santa Rosa e IAC-2. I - Efeitos sobre os parâmetros de produção. Planta Daninha, v. 6, n. 2, p. 86-100, 1983.

EMPRESA BRASILEIRA DE PESQUISA AGROPECUÁRIA - EMBRAPA. Recomendações técnicas para a cultura da soja na Região Central do Brasil 1998/ 1999. Londrina: 1998. 182 p. (EMBRAPA-CNPso. Documentos, 120).

GAZZIERO, D. L. P. et al. Levantamento preliminar da ocorrência de picão-preto (Bidens pilosa e Bidens subalternans) em áreas de cultivo de soja no Estado do Paraná. Inf. Ci. Plantas Daninhas, n. 2, v. 4, p. 4-5, 1998.

GUTIERRI, M. J. et al. DNA sequence variation in domain A of the acetolactate synthase genes of herbicide-resistant and susceptible weed biotypes. Weed Sci., v. 40, p. 670676, 1992.

HOLT, J. S.; LeBARON, H. M. Significance and distribution of herbicide resistance. Weed Technol., v. 4, n. 1, p. 141-149, 1990.

KISSMANN, K. G.; GROTH, D. Plantas infestantes e nocivas. 2.ed. São Paulo: BASF, 1999. 978 p. T. II.

MATIELLO, R. R.; RONZELI JÚNIOR, P.; PURÍSSIMO, C. Mecanismos de resistência: fatores biológicos, agronômicos e genéticos. In: CURSO DE MANEJO DA RESISTÊNCIA DE PLANTAS DANINHAS AOS HERBICIDAS, 2., Ponta Grossa, 1999. Anais... Ponta Grossa: AECG, 1999. p. 27-40.

MELHORANÇA, A. L.; VALENTE, T. O.; PEREIRA, F. A. Plantas daninhas. In: Soja: recomendações técnicas para Mato Grosso do Sul e Mato Grosso. Dourados: CPAO, 1996. p. 86-106. (EMBRAPA-CPAO, Circular Técnica, 3). 
MELO FILHO, G. A.; RICHETTI, A.; PARIZOTO, A. M. Aspectos socioeconômicos da cultura da soja. In: RECOMENDAÇÕES técnicas para Mato Grosso do Sul e Mato Grosso. Dourados: CPAO, 1996. p. 29-33. (EMBRAPA-CNPAO, Circular Técnica, 3).

PEREIRA, F. A. R.; MELHORANÇA, A. L.; BORGES, E. P.; VALENTE, T. O.; BAZONI, R. Herbicidas recomendados para a cultura da soja em Mato Grosso do Sul no ano agrícola 1999/2000. Campo Grande:

EMPAER-MS, 1999. 29 p. (Comunicado Técnico, 27).

PITELLI, R. A. Interferência de plantas daninhas em culturas agrícolas. Inf. Agropec., v. 11, n. 129, p. 16-27, 1985.

POWLES, S. B.; HOWAT, P. D. Herbicide-resistant weeds in Australia. Weed Technol., v. 4, n. 1, p. 178-185, 1990.
RODRIGUES, B. N.; ALMEIDA, F. S. Guia de herbicidas. 4.ed. Londrina: Edição dos Autores, 1998. 648 p.

SANCHES, W.; ZANDONADE, D. Problemas e soluções no controle de plantas daninhas no MS e MT (Relato n. 1). In: SIMPÓSIO SOBRE HERBICIDAS E PLANTAS DANINHAS, 1., 1997, Dourados. Resumos... Dourados: EMBRAPA-CPAO, 1997. p. 160-161. (EMBRAPA-CPAO. Documentos, 13).

VARGAS, L. et al. Resistência de plantas daninhas a herbicidas. Viçosa: Jard Produções Gráficas, 1999. 131 p.

VIDAL, R. A.; FLECK, N. G. Análise do risco da ocorrência de biótipos de plantas daninhas resistentes aos herbicidas. Planta Daninha, v. 15, n. 2, p. 152-161, 1997. 